\title{
Molecular (ISSR, cp DNA, ITS) and Morphological Study of the Genus Tragopogon L. (Asteraceae)
}

Hejraneh Azizi ( $\nabla$ hejraneh.azizi@yahoo.com )

Shahid Beheshti University

Masoud Sheidai

Shahid Beheshti University

Valiollah Mozaffarian

Research Institute of Forests and Rangelands

Zahra Noormohammadi

Azad University: Islamic Azad University

\section{Research Article}

Keywords: Tragopogon, Morphometry, ISSR, ITS, cp DNA.

Posted Date: January 10th, 2022

DOI: https://doi.org/10.21203/rs.3.rs-1160711/v1

License: (c) (i) This work is licensed under a Creative Commons Attribution 4.0 International License. Read Full License 
2 Molecular (ISSR, cp DNA, ITS) and Morphological study of the genus

3 Tragopogon L. (Asteraceae)

4

5

$6{\text { Hejraneh } \text { Azizi }^{1 *} \text {, Masoud Sheidai }}^{1}$, Valiollah Mozaffarian ${ }^{2}$, Zahra Noormohammadi ${ }^{3}$

7

$8 \quad{ }^{1}$ Faculty of life Sciences and Biotechnology, Shahid Beheshti University, Tehran, Iran

$9 \quad{ }^{2}$ Research Institute of Forests and Rangelands, Tehran, Iran

$10{ }^{3}$ Department of Biology, School of Basic Sciences, Science and Research Branch, Islamic Azad

11 University (SRBIAU), Tehran, Iran

$12 \quad{ }^{*}$ Corresponding author. E-mail: Hejraneh.azizi@yahoo.com

13

14

15

16

17

18

19

20

21

22

23

24 


\section{Abstract}

27 Tragopogon L. (Cichorioideae, Lactuceae, Scorzonerinae) is an Old World genus with 150 species, Rechinger in 28 Flora Iranica divided this genus in 13 section and 37 species that 26 species of them are exist in Iran. Safavi et al. 29 divided it into 26 species without sections in flora Iran. Despite the anatomical and molecular studies done around the 30 world, the exact classification of this genus is not clear due to the high number of secret species, hybridization, 31 polyploidy and rapid diversification. The morphology studies of 32 species and Molecular studies (ISSR, ITS, cp 32 DNA) of 22 species of the genus Tragopogon was investigated. The purpose of these studies are classification and 33 determination of interspecific relationship in this genus. Sections of Rubriflori, Sosnowskya,Chromopappus,Majores, 34 Angustissimi, Krascheninnikovia in flora of Iranica are confirmed on the basis of morphometry and molecular data. 35 Section of Profundisulcati in flora Iranica is confirmed on the base of morphometry data. The Species of T.jesdianus, 36 T.porphyrocephalus, T. rezaiyensis and T. Stroterocarpus in the flora of Iranica are not classified inanysectionwhich 37 we classified in the Rubriflori section, Cp DNA dendrogram are not useful for classification in this genus and 38 Chloroplast sequences are very similar among Tragopogon species, Therefore, the use of cp DNA markers in the 39 classification of this genus is not recommended. 


\section{Introduction:}

52 Tragopogon L. is an Old World genus of approximately 150 species that occurs across Eurasia from the Atlantic to the Pacific Ocean, with a center of distribution in the Mediterranean region, the Middle East and Eastern Europe

54 (Mavrodiev et al. 2005; 2008a-c). The monophyly of the genus was strongly supported in a recent phylogenetic 55 analysis of Scorzonerinae based on internal transcribed spacer (ITS) sequence data (Mavrodiev et al. 2004). Tragopogon includes biennial and perennial herbs with linear or linear-lanceolate leaves; solitary, simple, or sparingly branched stems; one or only a few capitulum and receptacles without scales. The achenes of Tragopogon are usually fusiform, with five to 10 more or less distinct ribs and a beak of varying length. The involucral bracts are always in one row, ligulate flowers are yellow or purplish, and the pappus is in one row of mostly plumose hairs (Richardson 1976). It is almost impossible to identify the species without adequate knowledge of mature achene and ligule color. Tragopogon is taxonomically difficult due to its high degree of species morphological variability, which in turnarises due to frequent occurrence of inter-specific hybridization and different ploidy levels (Mavrodievet al. 2008; Bell et al. 2012). Occurrence of interspecific hybridization reported in the literature (Ownbey 1950; Ownbey \& McCollum 1953; Pires et al. 2004; Buggs et al.2008; Mavrodievet al.2008) or facultative apomixis recently found in T. dubius (Kashin et al.2007)Relationships within Tragopogon are poorly understood. Many species of Tragopogon have not been placed in a section; most of these are narrow endemics that have been recognized and named but not treated taxonomically furthermore, the genus has never been the subject of a comprehensive monograph. Regional floras have provided treatments for species only in those geographic areas. (Mavrodiev et al. 2005). (Blanca and De la guardia, 1997) Schishkin (1961) In KomarovFlora Russia divided it in 17 section and 79 species that 10 species of them are share with flora Iran. Rechinger (1977) according to schishkin in flora Iranica divided this genus in 13 section and 37

71 species that 26 species (11 species endemic) of them are exist in Iran (Rechinger 1977). Safavi et al. (2014) divided 72 it into 26 species without sections in flora Iran. chloroplast DNA) sequence data. 
Materials and methods:

\section{Plant material}

Tragopogon species were collected from natural habitats in Iran in 2015-2018. The voucher specimens are deposited in the Shahid Beheshti University herbarium (HSBU), herbarium of Research Institute of Forests and Rangeland (TARI) and herbarium of Museum of Natural History Vienna (W). (Table 1)

\section{Morphological studies}

26 species of this genus belonging to the flora of Iran and 6 species belonging to the flora of Iranica were studied. Morphological characters studied were: Flower color, Anther tube color, Collar type, Investigation of branching in plant, presence of indumentums in plant, indumentums of involucre bracts, Number of flowers per pile, status of involucre bracts and ligule, Ratio length of bract to papus, shape of base leaves, Presence or absence of sheath in leaf, condition of tips of stem leaves, Leaf margin type, Leaf type, Condition of middle vein thickness, Central achene surface, Marginal achene surface, papus status, condition of papus and achene, papus type, Condition of beak to achene, beak type, condition of tip of beak, Surface on the beak, diameter of inflorescence, length of involucre, Width of involucre, length of bract, width of bract, number of involucre bracts, length of achene, width of achene, length of papus, length of beak, Number of vine or wings on the achene, length of the base leaves, width of the base leaves, Stem leaf length, stem leaf width, Number of basal leaf veins, ratio of length to width of the involucre, ratio of length to width of the bract, ratio of length to width of the achene, ratio of length to width of the papus, ratio of length to width of the beak, ratio of length to width of the base leaf, ratio of length to width of the stem leaf.

\section{DNA extraction and molecular assays}

Fresh leaves were collected from randomly selected plants and dried in silica gel powder. Genomic DNA was extracted using a CTAB activated charcoal protocol (Sheidai et al. 2013). The quality of extracted DNA was examined by electrophoresis on $0.8 \%$ agarose gels. four ISSR primers custom synthesized by UBC (the University of British Columbia) were used: UBC807, UBC810, UBC 834, (AGC) 5GA. PCR reactions were performed in a $25 \mu 1$ volume containing at final concentrations $10 \mathrm{mM}$ Tris- $\mathrm{HCl}$ buffer at $\mathrm{pH} 8,50 \mathrm{mM} \mathrm{KCl}, 1.5 \mathrm{mM} \mathrm{MgCl} 2,0.2 \mathrm{mM}$ of each dNTP (Bioron, Germany), $0.2 \mu \mathrm{M}$ of a single primer, $20 \mathrm{ng}$ genomic DNA and $3 \mathrm{U}$ of Taq DNA polymerase (Bioron, Germany). The DNA amplification reactions were performed in a thermocycler (Germany) with the following 
program: 5 min initial denaturation step at $94^{\circ} \mathrm{C}, 45 \mathrm{~s}$ at $94^{\circ} \mathrm{C} ; 1 \mathrm{~min}$ at $50^{\circ} \mathrm{C}$ and $1: 30 \mathrm{~min}$ at $72^{\circ} \mathrm{C}$. The reaction was completed with a 7 min extension step at $72^{\circ} \mathrm{C}$. The amplification products were visualized by electrophoresis on $2 \%$ agarose gels, followed by the ethidium bromide staining. The fragment size was estimated by using a 100 bp molecular size ladder (Fermentas, Germany).

The ITS5 region was amplified with $0.2 \mu \mathrm{M}$ primer ITS5 (5'-GGA AGT AAA AGTCGT AAC AAG G-3'; Bioron, Germany), and primer ITS4 (5'-TCC TCC GCT TATTGA TAT GC -3') (White et al. 1990). PCR reactions were performed in a $25 \mu \mathrm{l}$ volume containing at final concentration $10 \mathrm{mM}$ Tris- $\mathrm{HCl}$ buffer at $\mathrm{pH} 8,50 \mathrm{mM} \mathrm{KCl,} 1.5 \mathrm{mM}$ $\mathrm{MgCl} 2,0.2 \mathrm{mM}$ of each dNTP (Bioron, Germany), $20 \mathrm{ng}$ genomic DNA and $3 \mathrm{U}$ of Taq DNA polymerase (Bioron, Germany). The following thermocycler parameters were used: $95^{\circ} \mathrm{C}$ for $2 \mathrm{~min}$, followed by 33 cycles at $95^{\circ} \mathrm{C}$ for $30 \mathrm{~s}$, $56^{\circ} \mathrm{C}$ for $60 \mathrm{~s}$, and $72^{\circ} \mathrm{C}$ for $2 \mathrm{~min}$, followed by one final extension step at $72^{\circ} \mathrm{C}$ for $7 \mathrm{~min}$.

The plastid intergenic spacer psbA-trnH(GUG) was amplified and sequenced with universal primers following the methodology of Shaw et al. (2005) and Timme et al. (2007). The psbA-trnHGUG forward primer was trnHGUG (5'CGC GCA TGG TGG ATT CAC AAT CC-3') and, the reverse primer was psbA (5’- GTT ATG CAT GAA CGT AAT GCT C-3') (Table 2). Each $20 \mu \mathrm{l}$ PCR reaction contained $10 \mu \mathrm{l}$ of 2x PCR buffer, $0.5 \mathrm{mM}$ of each primer, 200 $\mathrm{mM}$ of each dNTP, 1 Unit of Taq DNA polymerase (Bioron, Germany), and $1 \mu$ l of template genomic DNA at 20 ng $\mu 1$ - 1 . The amplifications were performed in a Techne thermocycler (Germany) with the following program: 2 min initial denaturation step $94^{\circ} \mathrm{C}, 1 \mathrm{~min}$ at $94^{\circ} \mathrm{C} ; 1 \mathrm{~min}$ at $58^{\circ} \mathrm{C}$ and $1 \mathrm{~min}$ at $72^{\circ} \mathrm{C}$. The reaction was completed by a final extension step of $6 \mathrm{~min}$ at $72^{\circ} \mathrm{C}$.

The PCR products were electrophoresed on 2.5\% agarose gels and visualized through GelRed ${ }^{\mathrm{TM}}$ Nucleic Acid Gel Staining. Fragment size was estimated using a 100 bp size ladder (Thermo- Fisher Scientific, Waltham, MA USA).

\section{Data analysis}

\section{Morphological analyses}

Grouping of the species was obtained by using UPGMA (Unweighted Paired Group Method with Arithmetic mean) and Ward (Minimum spherical cluster method) as well as PCoA (Principal Coordinate Analysis) (Podani, 2000). Morphological characters were first standardized $($ Mean $=0$, Variance $=1)$ and used to establish Euclidean distances among pairs of taxa (Podani, 2000; Sheidai et al., 2014). The obtained distances were used for clustering. 
Morphological difference of the studied species was investigated by ANOVA (Analysis of Variance) and CVA (Canonical Variance Analysis). PCA (Principal Components Analysis) was performed to identify the most variable morphological characters (Podani, 2000). Morphometric analyses were performed by PAST ver. 2.17 (Hammer et al., 2012).

\section{ISSR analysis}

Significances of genetic differences among the studied species was determined by AMOVA(Analysis of Molecular Variance) with 1000 permutations for dominant molecular markers as implemented in GenAlex v.6.4 (Peakall and Smouse 2006). Nei's genetic distances were determined among the studied species and used for clustering. For grouping of the plant specimens, Neighbor Joining (NJ) clustering and PCoA were used (Podani 2000). GenAlex 6.4, and PAST v.2.17 (Hammer et al. 2012), programs were used for these analyses. The genetic structure of species was studied by STRUCTURE (Pritchard et al. 2000), for dominant markers (Falush et al. 2007), using the admixture model. The Markov chain Monte Carlo simulation was run 20 times for each value of K for 106 iterations after a burnin period of 105. All other parameters were set at their default values.

\section{Cp-DNA and nuclear gene sequence analyses}

The intergenic chloroplast spacer psbA-trnHGUG as well as the ITS region nuclear sequences obtained for all the studied species. The sequences obtained were aligned by MUSCLE program implemented in MEGA ver. 5 (Tamura et al. 2011) and used to study the species relationship by different phylogenetic reconstruction methods like: Neighbor Joining, UPGMA clustering (Unweighted paired group using average), and maximum parsimony and maximum likelihood using MEGA v. 5 (Tamura et al. 2011) The proper model for sequence evolution was determined by the same option provided in MEGA program. 
Table 1. Tragopogon species studied, their locality information, code and voucher number

\begin{tabular}{|c|c|c|c|c|}
\hline & Species & code & Locality & Voucher \\
\hline 1 & T. capitatus & Cap & Iran: East Mazandaran & W-2000/06412 \\
\hline 2 & T. vaginatus & Vag1 & Iran: West Azerbaijan: Sardasht- Piranshahr(IS) & TARI-7748 \\
\hline 2 & T. vaginatus & Vag2 & Iran: West Azerbaijan: Ardalan village (IS) & TARI-65501 \\
\hline 2 & T. vaginatus & Vag3 & Iran: Khalkhal to Ardebil & TARI-86534 \\
\hline 2 & T. vaginatus & Vag4 & Iran: East Azerbaijan: Sahand (IS) & TARI-30669 \\
\hline 2 & T. vaginatus & Vag5 & Iran: Kurdistan: Karabad (IS) & TARI-644 \\
\hline 2 & T. vaginatus & Vag6 & Iraq: Sulaimaniya, inte Sulaimaniya et Dokan & W-1991/4989 \\
\hline 2 & T. vaginatus & Vag7 & Iraq: Sulaimaniya, inter Kirkuk to ward Sulaimaniya & W- 1991/19529 \\
\hline 3 & T. afghanicus & Afg1 & Afghanestan: qala salcan & W- $1956 / 3032$ \\
\hline 3 & T. afghanicus & $\operatorname{Afg} 2$ & afghanestan: Khurak & W-1956/3122 \\
\hline 4 & T. paradoxus & Par1 & $\begin{array}{l}\text { Afghanestan: N Darai suf, between qala sarkari and } \\
\text { kupruk }\end{array}$ & W-1987/4287 \\
\hline 4 & T. paradoxus & Par2 & Afghanestan: nordl. Pul e khomrie & W-1965/15880 \\
\hline 5 & T. kemulariae & Kem1 & Iran: West Azerbaijan: Khoy, Qoture (IS) & TARI-84101 \\
\hline 5 & T. kemulariae & Kem2 & Iran: Hamadan: Fakhr abad (IS) & TARI-4254 \\
\hline 5 & T. kemulariae & Kem3 & Iran: Kurdistan: 20 km toward Hamadan (IS) & TARI-84907 \\
\hline 5 & T. kemulariae & Kem4 & Iran: Weast Azerbaijan: Khoy, Grees village (IS) & TARI-84132 \\
\hline 5 & T. kemulariae & Kem5 & Iran: Ardebil: Arasbaran (IS) & TARI-24712 \\
\hline 5 & T. kemulariae & Kem6 & Iran: Ardebil: Arasbaran (IS) & TARI-24651 \\
\hline 6 & T. sosnowsky & Sos & Armenia,Sevan & W-2004/2395 \\
\hline 7 & T. vvedenskyi & Vve1 & Afghanestan: Farakulum & W-1980/3311 \\
\hline
\end{tabular}




\begin{tabular}{|c|c|c|c|c|}
\hline 7 & T. vvedenskyi & Vv2 & Iran: Tehran: Haraz valley & TARI-85712 \\
\hline 7 & T. vvedenskyi & Vv3 & Iran: West Azerbaigan: Silvana to Salmas road (IS) & TARI-85193 \\
\hline 7 & T. vvedenskyi & Vv4 & Iran: Semnan & TARI-40684 \\
\hline 7 & T. vvedenskyi & Vv5 & Iran: Isfahan: Golestan koh (IS) & TARI-83087 \\
\hline 7 & T. vvedenskyi & Vv6 & Iran: Gorgan: Almeh & TARI-12618 \\
\hline 7 & T. vvedenskyi & Vv7 & Iran: Gorgan: Almeh & TARI-12619 \\
\hline 7 & T. vvedenskyi & Vv8 & Iran: Mazandaran: Plur to Rineh ( 4 IS)(cp)(ITS) & HSBU-2018800 \\
\hline 8 & T. longirostris & Long1 & Iran: Tehran: solghan valley & TARI-32617 \\
\hline 8 & T. longirostris & Lon2 & Iran: Khorasan: Tandureh,Chehel mehr & TARI-50839 \\
\hline 8 & T. longirostris & Long3 & Iran: Chahar mahale bakhtiari:Shalam zar,Agha saner & TARI-54586 \\
\hline 8 & T. longirostris & Long4 & Iran: West Azerbaigan: Urmieh lake,Islamic island & TARI-86916 \\
\hline 8 & T. longirostris & Long5 & Iran: Kermanshah & TARI-60769 \\
\hline 8 & T. longirostris & Long6 & Iran: Kurdistan: Baneh to Marivan & TARI-29317 \\
\hline 8 & T. longirostris & Long7 & Iran: Gorgan: Almeh & TARI- 12620 \\
\hline 8 & T. longirostris & Long8 & Iran: Fars:Nor abad & TARI-45696 \\
\hline 8 & T. longirostris & Long9 & Iran: Alborz: Karaj- Chalus (IS) & HSBU-2018801 \\
\hline 8 & T. longirostris & $\begin{array}{l}\text { Long1 } \\
0\end{array}$ & Iran: Kurdistan: Baneh & HSBU-2018802 \\
\hline 8 & T. longirostris & $\begin{array}{l}\text { Long1 } \\
1\end{array}$ & Iran: Kurdistan: Saqez, Zagheh (2 IS) & HSBU-2018803 \\
\hline 8 & T. longirostris & $\begin{array}{l}\text { Long1 } \\
2\end{array}$ & Syria * & W-1980/296324 \\
\hline 8 & T. longirostris & $\begin{array}{l}\text { Long1 } \\
3\end{array}$ & Iraq: Sulaimaniya, inter Kirkuk to ward Sulaimaniya & W-1980/19526 \\
\hline 8 & T. longirostris & $\begin{array}{l}\text { Long1 } \\
4\end{array}$ & E- Afghanestan: Kabul, Band-I Kharghak & W-1980/619 \\
\hline 9 & T. badakhshanicus & $\mathrm{Bad}$ & Afghanestan: Wakhan, Ab-Gaj & W-1965/18843 \\
\hline
\end{tabular}




\begin{tabular}{|c|c|c|c|c|}
\hline 10 & T. gracilis & Grac1 & Afghanestan: Paktya, Kotgay, Mandaher wald & W-1980/1725 \\
\hline 10 & T. gracilis & Grac2 & East Afghanestan: Khost, chakmanni & W-1980/777 \\
\hline 11 & T. gongylorrhizus & Gon1 & Iran: West Azerbaigan: Urmieh lake, Spireh island & TARI-15052 \\
\hline 11 & T. gongylorrhizus & Gon2 & Iran: Gorgan \# & TARI-15053 \\
\hline 11 & T. gongylorrhizus & Gon3 & Iran: Gorgan: Golestan Park (IS) & W-1980/5141 \\
\hline 11 & T. gongylorrhizus & Gon4 & Iran: Gorgan & TARI-5123 \\
\hline 12 & $\begin{array}{c}\text { T. buphthalmoides subsp. } \\
\text { buphthalmoides }\end{array}$ & Bupb1 & Iraq: Suleimaniya to Ghala Daze & W-1980/19531 \\
\hline 12 & $\begin{array}{c}\text { T. buphthalmoides subsp. } \\
\text { buphthalmoides }\end{array}$ & Bupb2 & Iran: Tehran: Dehnar(IS) & HSBU -4006 \\
\hline 12 & $\begin{array}{c}\text { T. buphthalmoides subsp. } \\
\text { buphthalmoides }\end{array}$ & Bupb3 & Iran: West Azerbaijan: Sardasht, Gerzhal(IS) & HSBU -4007 \\
\hline 12 & $\begin{array}{c}\text { T. buphthalmoides subsp. } \\
\text { buphthalmoides }\end{array}$ & Bupb4 & Iran: West Azerbaijan: Jaldian & HSBU -4008 \\
\hline 12 & $\begin{array}{c}\text { T. buphthalmoides subsp. } \\
\text { buphthalmoides }\end{array}$ & Bupb5 & Iran: Mazandaran: Siah bisheh(IS) & HSBU -4009 \\
\hline 12 & $\begin{array}{c}\text { T. buphthalmoides subsp. } \\
\text { buphthalmoides }\end{array}$ & Bupb6 & Iran: Mazandaran: Rineh & HSBU -4010 \\
\hline 12 & $\begin{array}{c}\text { T. buphthalmoides subsp. } \\
\text { buphthalmoides }\end{array}$ & Bupb7 & Iran: Alborez: Gach sar(IS) & HSBU -4011 \\
\hline 12 & $\begin{array}{c}\text { T. buphthalmoides subsp. } \\
\text { buphthalmoides }\end{array}$ & Bupb8 & Iran: Kurdistan: Kani bard & HSBU -4012 \\
\hline 12 & $\begin{array}{c}\text { T. buphthalmoides subsp. } \\
\text { buphthalmoides }\end{array}$ & Bupb9 & Iran: Isfahan: Naein (IS) & HSBU -4013 \\
\hline 12 & $\begin{array}{c}\text { T. buphthalmoides subsp. } \\
\text { buphthalmoides }\end{array}$ & $\begin{array}{l}\text { Bupb1 } \\
0\end{array}$ & Iran: Semnan & HSBU -4014 \\
\hline 12 & $\begin{array}{c}\text { T. buphthalmoides subsp. } \\
\text { Latifolius }\end{array}$ & Buphb11 & Iran: Kurdistan: Saqez & HSBU -4015 \\
\hline
\end{tabular}




\begin{tabular}{|c|c|c|c|c|}
\hline 12 & $\begin{array}{c}T . \\
\text { buphthalmoides } \\
\text { subsp. Latifolius }\end{array}$ & Bupl1 & Iran: Kurdistan: Boein (IS) & HSBU -4016 \\
\hline 12 & $\begin{array}{c}T . \\
\text { buphthalmoides } \\
\text { subsp. Latifolius }\end{array}$ & Bupl2 & Iran: Mazandaran: Rineh (IS) & HSBU -4017 \\
\hline 12 & $\begin{array}{c}T . \\
\text { buphthalmoides } \\
\text { subsp. Latifolius }\end{array}$ & Bupl3 & Iran: Alborez: Gachsar (IS) & HSBU -4018 \\
\hline 12 & $\begin{array}{c}T . \\
\text { buphthalmoides } \\
\text { subsp. Latifolius }\end{array}$ & Bupl4 & Iran: Tehran: Dehnar (IS) & HSBU -4019 \\
\hline 12 & $\begin{array}{c}T . \\
\text { buphthalmoides } \\
\text { subsp. Latifolius }\end{array}$ & Bupl5 & Iran: West Azerbaijan: Mosalan & HSBU-4020 \\
\hline 12 & $\begin{array}{c}T . \\
\text { buphthalmoides } \\
\text { subsp. Latifolius }\end{array}$ & Bupl6 & Iran: West Azerbaijan: Sardasht,Gerzhal (IS)(ITS) & HSBU-4021 \\
\hline 13 & T. rechingeri & $\operatorname{Rec} 1$ & Iran:(Hamadan: Agh blagh (IS) & TARI-3392 \\
\hline 13 & T. rechingeri & $\operatorname{Rec} 2$ & $\begin{array}{l}\text { Iran: Hamadan: } 20 \mathrm{~km} \text { Asad abad to Akhtachi (IS) } \\
\text { village }\end{array}$ & TARI-3443 \\
\hline 13 & T. rechingeri & $\operatorname{Rec} 3$ & $\begin{array}{l}\text { Iran: Hamadan: } 20 \mathrm{~km} \text { Asad abad to Akhtachi } \\
\text { village(IS) }\end{array}$ & TARI-3444 \\
\hline 13 & T. rechingeri & $\operatorname{Rec} 4$ & Iran: Hamadan:8 km East Ganj nameh (IS) & TARI-36848 \\
\hline 13 & T. rechingeri & $\operatorname{Rec} 5$ & Iraq: Kurdistan: Erbil, Haji omran * & W-1980/5240 \\
\hline 14 & $\begin{array}{l}\text { T. bornmuelleri } \\
\text { var. bornmuelleri }\end{array}$ & Borb1 & $\begin{array}{l}\text { Iran: Kurdistan: } 36 \text { km Sanandaj to Kamiaran, (2IS) } \\
\text { Noshor Valley }\end{array}$ & TARI-60592 \\
\hline 14 & $\begin{array}{l}\text { T. bornmuelleri } \\
\text { var. bornmuelleri }\end{array}$ & Borb2 & Iran: Kurdistan: Maran village (2IS) & TARI-60418 \\
\hline 14 & $\begin{array}{l}\text { T. bornтиelleri } \\
\text { var. bornmuelleri }\end{array}$ & Borb3 & Iran: Kurdistan: Saqez to Baneh (2 IS) & TARI-2951 \\
\hline
\end{tabular}


var. bornmuellerVai

T. bornmuelleri var. bornmuelleri

\section{T. bornmuelleri} var. latifolius

\section{T.}

\section{T. bornmuelleri} var. latifolius
Borb

Borl1

Iran: Kermanshah to Kamiaran (IS)(ITS)

TARI-87735

Bor12 Iran: Kermanshah:Mansure agha village, Shahoo

mountain

Iran: Mazandaran: Rineh, Abgarm

T. acanthocarpus Aca2 Iran: Zanjan: $10 \mathrm{~km}$ Mah neshan, Ghezal ozan river

Iran: East Azerbaijan: Marand to Jolfa, Zenoz, $22 \mathrm{~km}$

Zenoz to Kohe Kamar

T. acanthocarpus Aca4 Iran: West Azerbaijan: Urmieh University (IS)

T. acanthocarpus Aca5 Iran: Alborz: Karaj, Research center to Alborz

T. acanthocarpus Aca6 Iran: Hamadan: Absard (IS)

T. acanthocarpus Aca7 Iran: Markazi: Arak, Miran khaneh, Sefid khani (IS)

TARI-47745

T. acanthocarpus Aca8 Iran: Mazandaran: Dashte Nazir (IS)

HSBU-2018804

T. acanthocarpus Aca9 Iran: Kurdistan: Salavat abad village (IS)

HSBU-2018805

T. acanthocarpus Aca10 Iran: Tehran: Firuz koh (IS)

HSBU-2018806

T. graminifolius $\quad$ Gra1 Iran: Tehran: Chitgar (IS)

HSBU-14466

T. graminifolius Gra2 Iran: North Khorasan:Ziarat, Shirvan (IS)

HSBU-14466

T. graminifolius Gra3 Iran: East Azarbaijan: Ilkhchi (IS)

HSBU-14469

T. graminifolius Gra4 Iran: West Azarbaijan: Orumieh

T. graminifolius Gra5 Iran: Markazi: Arak, Gavkhaneh(IS)

T. graminifolius Gra6 Iran: Isfahan: Semirom (IS)
HSBU-14470

HSBU-14471

HSBU-14472 


\begin{tabular}{|c|c|c|c|c|}
\hline 17 & T. reticulatus & Ret1 & Iran: West Azarbaijan: Orumieh lake, Kabodan island & TARI-24916 \\
\hline 17 & T. reticulatus & Ret2 & Iran: Khozestan: Deh dez (IS) & TARI-63121 \\
\hline 17 & T. reticulatus & Ret3 & Iran: West Azarbaijan: Ghasemloo(IS) & TARI-4184 \\
\hline 17 & T. reticulatus & Ret4 & Iran: Hamedan: Abas abad(IS) & TARI-4252 \\
\hline 17 & T. reticulatus & Ret5 & Iran: kurdistan:baneh,boeen(IS) & TARI-2081 \\
\hline 17 & T. reticulatus & Ret6 & Iran: Mazandaran:Polor,Haraz Road & TARI-4461 \\
\hline 18 & T. kotschyi & Kot1 & \# & W-1986/32097 \\
\hline 18 & T. kotschyi & Kot2 & Iran: Mazandaran:91 Km Karaj to Chalous(IS) & TARI-5430 \\
\hline 18 & T. kotschyi & Kot3 & Iran: East Azerbayjan :Kandovan(IS) (cp) & TARI-4504 \\
\hline 18 & T. kotschyi & Kot4 & Iran: Tehran:gajereh(IS) & TARI-24193 \\
\hline 18 & T. kotschyi & Kot5 & Iran: Tehran:Gajereh(IS) & TARI-5473 \\
\hline 18 & T. kotschyi & Kot6 & Iran: Mazandaran:8 $\mathrm{km}$ to polor & TARI-86047 \\
\hline 18 & T. kotschyi & Kot7 & Iran: Gorgan:Aalmeh & TARI-12619 \\
\hline 18 & T. kotschyi & Kot8 & Iran: Lorestan:Khoram Abad(IS) & HSBU-2018813 \\
\hline 19 & T. marginatus & Mar1 & Iran: West Azarbayejan:20 Km to Seyah Rod & TARI-86729 \\
\hline 19 & T. marginatus & Mar2 & Iran: West Azarbayjan :Arasbaran,Topkhaneh & TARI-81373 \\
\hline 19 & T. marginatus & Mar3 & Iran: Karaj:Kondar(IS) & TARI-4374 \\
\hline 19 & T. marginatus & Mar4 & Iran: Yazd:Tarzjan Mountain,South Eastern Shirkoh & TARI-1452 \\
\hline 19 & T. marginatus & Mar5 & Iran: West Azarbayjan: Uromieh,Sero Road(IS) & TARI-7708 \\
\hline 19 & T. marginatus & Mar6 & Iran: Ardabil:Arasbaran(IS) & TARI-20954 \\
\hline 19 & T. marginatus & Mar7 & Iran: West Azarbayjan:Sahand Mountain(IS) & TARI-99404 \\
\hline 19 & T. marginatus & Mar8 & Iran: Gilan:Damash to Jirandeh & TARI-13515 \\
\hline 20 & T. maturatus & Mat & Iran: Golestan:Golestan jungle,Tangeh Rah & W-1976/10148 \\
\hline 21 & T. coloratus & Colo1 & Iran: Gorgan & TARI-296322 \\
\hline
\end{tabular}




\begin{tabular}{|c|c|c|c|c|}
\hline 21 & T. coloratus & Colo2 & $\begin{array}{l}\text { Iran: Gorgan:18 Km from Maraveh Tapeh to Ash } \\
\text { Khaneh }\end{array}$ & TARI-55598 \\
\hline 21 & T. coloratus & Colo3 & Iran: Gorgan:43 Km Rodbar to Gilan & TARI-60180 \\
\hline 21 & T. coloratus & Colo4 & Iran: Azarbayjan:30 Km Southern Khalkhal(IS) & TARI-36251 \\
\hline 21 & T. coloratus & Colo5 & Iran: Zanjan:25 $\mathrm{Km}$ from Gilvan to Zanjan & TARI-60280 \\
\hline 21 & T. coloratus & Colo6 & Iran: Tehran:Sorkheh Hesar(IS) & TARI-5476 \\
\hline 21 & T. coloratus & Colo7 & Iran: Tehran:Hesarak & TARI-5481 \\
\hline 21 & T. coloratus & Colo8 & Iran: Arak:Miran house,Kooh Sefid Khani(IS) & TARI-47748 \\
\hline 21 & T. coloratus & Colo9 & $\begin{array}{l}\text { Iran: Kermanshah:Kamyaran,Varmanjeh,Padegan (IS) } \\
\text { Shahid Rajaee }\end{array}$ & TARI-87737 \\
\hline 21 & T. coloratus & Colo10 & Iran: Kermanshah:Tagh Bostan,Tangeh Konesht(IS) & TARI-87738 \\
\hline 21 & T. coloratus & Colo11 & $\begin{array}{l}\text { Iran: Ardabil:Arasbaran protect region,Shib (IS) } \\
\text { Jonobi Jangal Tahghighati }\end{array}$ & TARI-81348 \\
\hline 22 & T. pterocarpus & Pte1 & Iran: Chahar Mahal Bakhtyari:Shahr Kord,Shams Abad & TARI-96641 \\
\hline 22 & T. pterocarpus & Pte2 & $\begin{array}{l}\text { Arak:Arak to Khomeyn,Koh Vercheh to Istgah } \\
\text { Macrowave Iran: }\end{array}$ & TARI-48044 \\
\hline 22 & T. pterocarpus & Pte3 & $\begin{array}{l}\text { Iran: Ghazvin:Almot Region,Balaye Rostaye Moalem } \\
\text { Kalateh }\end{array}$ & TARI-50992 \\
\hline 22 & T. pterocarpus & Pte4 & Iran: Alborz:40 Km Karaj Chalos,Seyeda Chal & TARI-5447 \\
\hline 22 & T. pterocarpus & Pte5 & Iran: Ardabil:8 Km north Khlkhal,Kelar Abad Road & TARI-34091 \\
\hline 22 & T. pterocarpus & Pte6 & Iran: Isfahan:Ghamishlo protect region,Khersak(IS) & TARI-1074 \\
\hline 22 & T. pterocarpus & Pte7 & $\begin{array}{l}\text { Iran: Kurdistan:36km Sanandaj to Kamyaran, (IS) } \\
\text { noshur valley }\end{array}$ & TARI-60624 \\
\hline 22 & T. pterocarpus & Pte8 & Iran: West Azarbaijan: Khoy, Razy valley(IS) & TARI-1392 \\
\hline 22 & T. pterocarpus & Pte9 & Iran: Semnan: Abr jungle (IS) & HSBU-2018814 \\
\hline 22 & T. pterocarpus & Pte 10 & Iran: Tehran: Chaloos road, Moroud (IS) & HSBU-2018815 \\
\hline 23 & T. collinus & Coli1 & Iran: Gilan: between Gazvin and Rudbar & TARI-27662 \\
\hline
\end{tabular}




\begin{tabular}{|c|c|c|c|c|}
\hline 23 & T. collinus & Coli2 & Iran: Khorasan: Birjand, Western part, Asfaraz village & TARI-83349 \\
\hline 23 & T. collinus & Coli3 & Iran: Khorasan: 42 km Birjand & TARI-84745 \\
\hline \multirow[t]{2}{*}{23} & T. collinus & Coli4 & Iran: Khorasan: Birjand, around Sar bishe, (IS) & TARI- 83340 \\
\hline & & & Salmabad & \\
\hline 23 & T. collinus & Coli4 & Iran: Tehran: Saveh, Rud shor (IS) & TARI-9632 \\
\hline 23 & T. collinus & Coli5 & Iran: Tehran: Saveh, Rud shor(IS) & TARI-9633 \\
\hline 23 & T. collinus & Coli6 & Iran: Isfahan: Semirom, Abshar (IS) & TARI-88600 \\
\hline 23 & T. collinus & Coli7 & Iran: Isfahan: Semirom, Abshar(IS) & TARI-88601 \\
\hline 24 & T.caricifolius & Car1 & Iran: Tehran: Homand, Absard & TARI-5465 \\
\hline 24 & T.caricifolius & Car2 & Iran: Mazandaran: Chalus, Dashte Nazir(IS) & TARI-1377 \\
\hline 24 & T.caricifolius & Car3 & Iran: Karaj: 12 km northwest Karaj & TARI-5470 \\
\hline 24 & T.caricifolius & Car4 & Iran: East Azarbaijan: $20 \mathrm{~km}$ Tabriz to Tehran & TARI-28013 \\
\hline 24 & T.caricifolius & Car5 & Iran: Yazd: 50 km east Bafegh, Hamsuk village(IS) & TARI-56083 \\
\hline 24 & T.caricifolius & Car6 & Iran: Bandar Abas: Geno mountain & TARI-16083 \\
\hline 24 & T.caricifolius & Car7 & Iran: Kerman: 50 kmWest of Khajeh Mountain (IS) & TARI-56206 \\
\hline 24 & T.caricifolius & Car8 & $\begin{array}{l}\text { Iran: Baluchestan: Taftan mountain, Tamndan (IS) } \\
\text { region }\end{array}$ & TARI-53202 \\
\hline 24 & T.caricifolius & Car9 & Iran: Tehran: Darband sar(IS) & TARI-49230 \\
\hline 25 & T. bakhtiaricus & Bak1 & $\begin{array}{l}\text { Iran: Chahar mahale Bakhtiari: Brojen, Research } \\
\text { Institute of Forests and Rangelands (IS)(cp) }\end{array}$ & TARI-54377 \\
\hline 25 & T. bakhtiaricus & Bak2 & $\begin{array}{l}\text { Iran: Chahar rmahale Bakhtiari: Brojen, Bar aftab (IS) } \\
\text { mountain }\end{array}$ & TARI-54767 \\
\hline 25 & T. bakhtiaricus & Bak3 & Iran: Fars: Kharman mountain(IS) & TARI-46922 \\
\hline 25 & T. bakhtiaricus & Bak4 & $\begin{array}{l}\text { Iran: Chahar mahale Bakhtiari:Vardanjan to (IS) } \\
\text { Kakolak }\end{array}$ & TARI-54126 \\
\hline 25 & T. bakhtiaricus & Bak5 & Iran: Gilan: Loshan to Amar loo(IS) & TARI-5445 \\
\hline 26 & T. gaudanicus & Gaul & Iran: Fars: Kazeron, ketel (pir zan)(IS) & TARI-9145 \\
\hline
\end{tabular}


Gau5

Afghanestan: Abe chist

W-1980/3031

Bojnord

T. montanus Mon

Iran: Golestan: Almeh jungle (IS)

TARI-4285 mountain abad

TARI-28953 


\begin{tabular}{|c|c|c|c|c|}
\hline 29 & T. jesdianus & Jez9 & $\begin{array}{l}\text { Iran: Semnan: } 30 \mathrm{~km} \text { northern west Shahrud, (IS) } \\
\text { between Tash and Chahar bagh }\end{array}$ & TARI-21160 \\
\hline 30 & $\begin{array}{l}T . \\
\text { porphyrocephalus }\end{array}$ & Pro1 & Iran: West Azarbaijan: Rezaeieh lake & W-1964/4029 \\
\hline 30 & $\begin{array}{c}T . \\
\text { porphyrocephalus }\end{array}$ & Pro2 & $\begin{array}{l}\text { Iran: Chahar mahale Bakhtiari: Shahre kord, (IS) } \\
\text { Harchegan }\end{array}$ & TARI-62060 \\
\hline 30 & $\begin{array}{l}\text { T. } \\
\text { porphyrocephalus }\end{array}$ & Pro3 & $\begin{array}{l}\text { Iran: Kurdistan: } 40 \text { km north Kamyaran to (IS) } \\
\text { Sanandaj, Faghieh Soleiman }\end{array}$ & TARI-370 \\
\hline 31 & T. rezaiyensis & Rezl & $\begin{array}{l}\text { Iran: Khorasan: Torbate Heidarieh to Mashhad, } 5 \mathrm{~km} \\
\text { Kameh Sofla }\end{array}$ & TARI-84842 \\
\hline 31 & T. rezaiyensis & Rez2 & Iran: Tehran: Gilavand & HSBU-2018816 \\
\hline 31 & T. rezaiyensis & Rez3 & Iran: Tehran: Shahid Beheshti university & HSBU-2018817 \\
\hline 31 & T. rezaiyensis & Rez4 & Iran: Tehran: Abali & HSBU-2018818 \\
\hline 31 & T. rezaiyensis & Rez5 & Iran: West Azarbaijan: Rabus valley to Rezaeieh & W-2000/5182 \\
\hline 32 & T. stroterocarpus & Str & Iran: West Azarbaijan: Sardasht & W-1980/5183 \\
\hline
\end{tabular}

\section{$158 \quad$ Results}

159 Morphometry

161 UPGMA, NJ dendrogram, Ward and PCA plot and Parsimony produced similar results therefore, only UPGMA 162 dendrogram is presented here (Fig 1). species delimitation between the studied species are determinedfromeachother, T. capitatus ‘T. vaginatus 'T. afghanicus ‘T. paradoxus located in one cluster Which T. capitatus, T. afghanicus and

164 T. paradoxus belong to the flora of Iranica and do not exist in Iran. Populations belonging to T. kemulariae together, populations belonging to T. longirostris together and populations belong to T. gngylorhizus to gether have formed cluster. 
167

168

169

170

171

172

173

174

175

176

177

178

179

180

181

182

183

184

185

186

187

188

189

190

191
T. badachschanicus and T. gracilis species and T. sosnovsky and T. vvedenskyi species show the closest relationship with each other. The species T. badachschanicus, T. gracilis and T. sosnovsky do not exist in Iran and belong to the flora of Iranica.

T. kotschy, T. marginatus and one population of T. reticulatus together in a cluster and populations of T. collinus, T. caricifolius, T. bakhtiaricus, T. gaudanicus and one population of $T$. montanus are located in the same cluster. The populations of T. buphthalmoides, T. bornmuelleri, T. rechingeri, T. acanthocarpus, T. graminifolius with five populations of $T$. reticulatus are in the same cluster. The populations of $T$. pterocarpus and T. coloratus are locatedin two separated clusters and are located close to each other.

PCA analysis of morphological characters revealed that the firs 3 PCA components comprise about $76 \%$ of total variability. Diameter of inflorescence, flower color, number of bract and length of beak showed the highest level of correlation with the first PCA component (40), while character length of papus, length of achene, status of involucre bracts and ligule were highly correlated with the second PCA component (12.81). Therefore, these are the most variable morphological characters among the studied species.

80

\section{1}

82

83




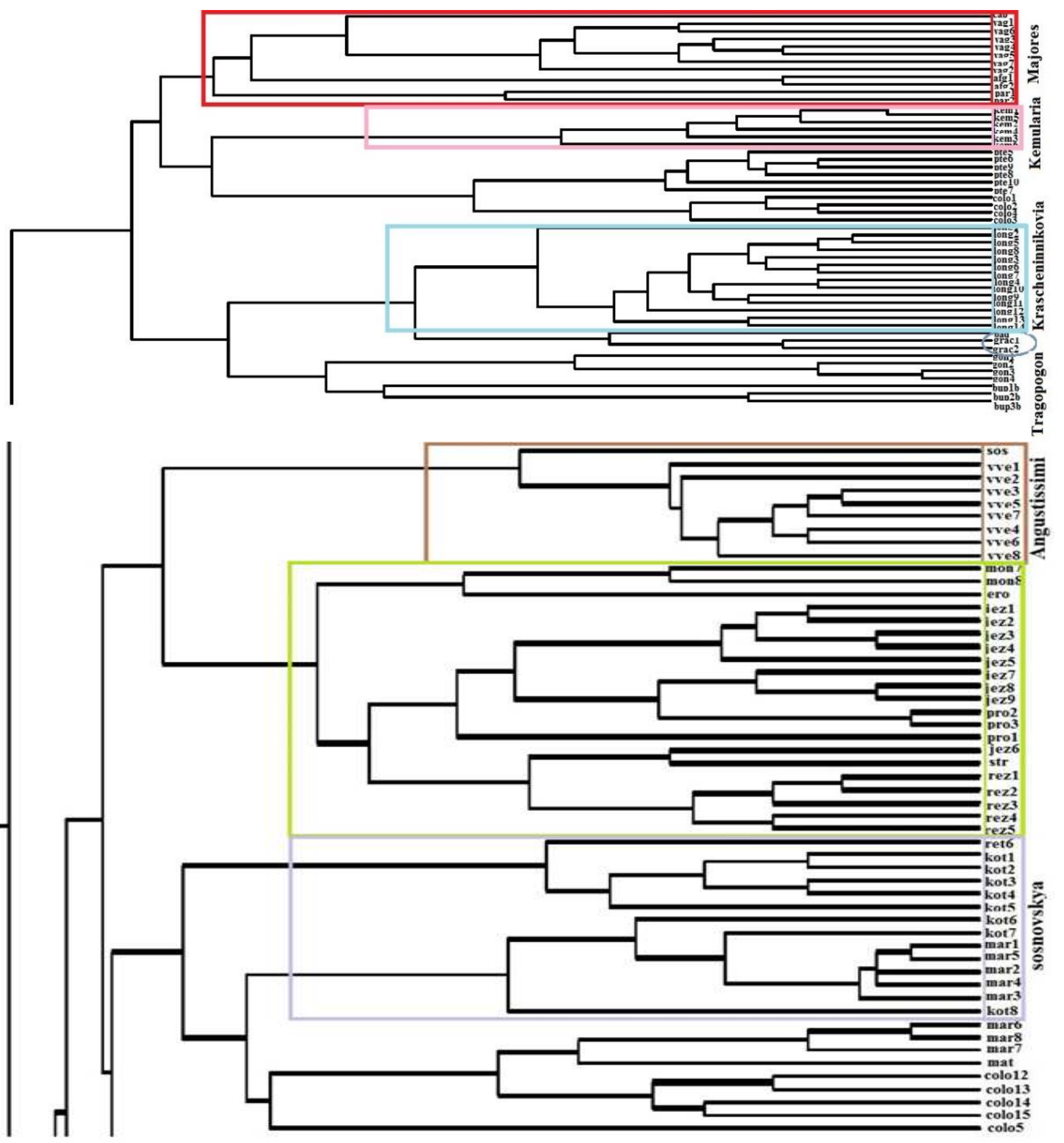




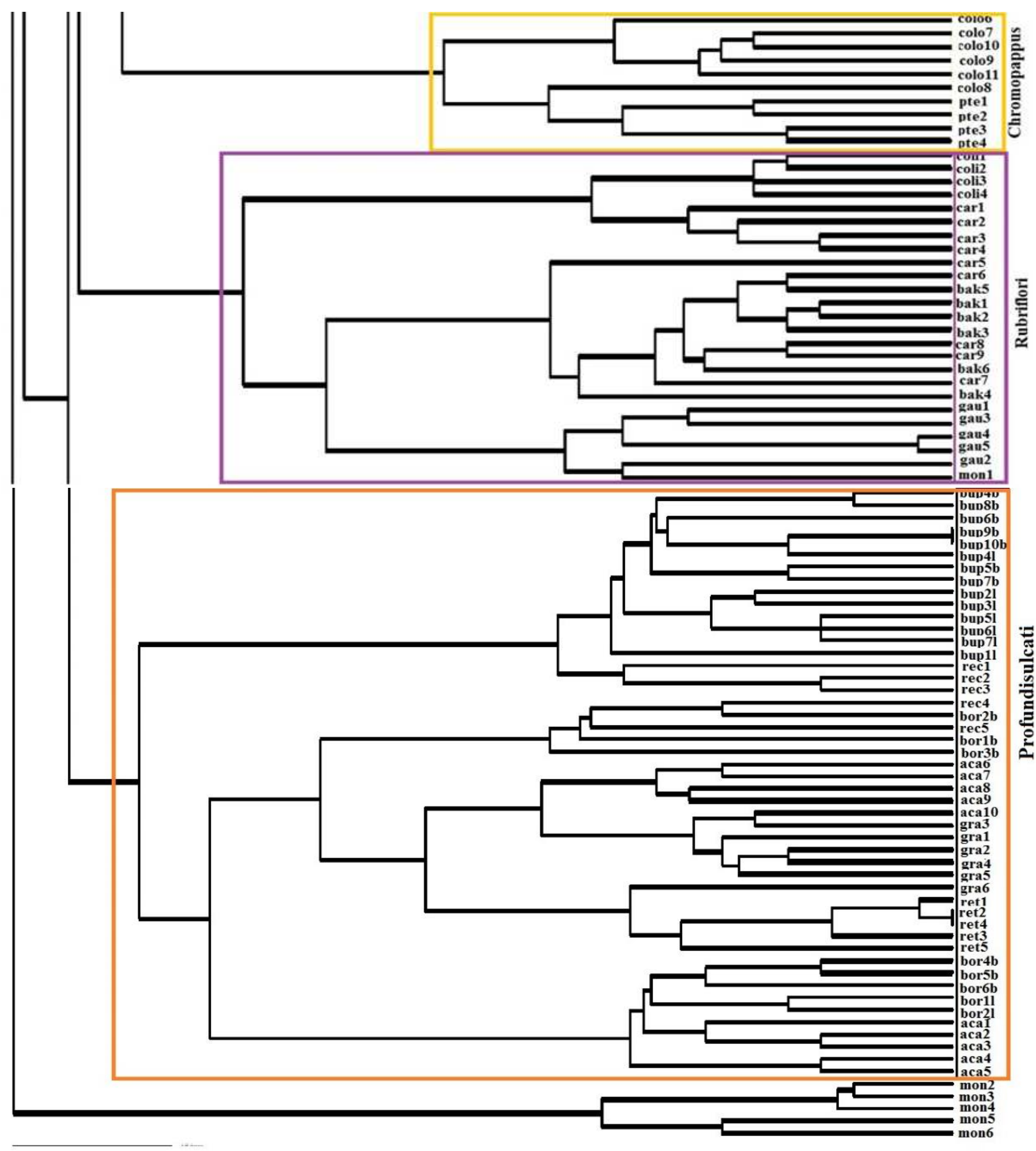


Fig 1. UPGMA dendrogram of morphological characters among Tragopogon species (Species code are according to 195 Table 1).

\section{ISSR markers}

198 This study was performed on 110 individuals belonging to 102 populations of 22 species of this genus. We obtained 199153 reproducible ISSR bands from almost all ISSR primers used. These bands formed our initial data matrix. AMOVA test revealed the presence of significant molecular difference among the studied populations $(\mathrm{P}=0.01)$. It also revealed that $60 \%$ of total variance occurred due within species genetic variability, while $40 \%$ was due to among species genetic difference. UPGMA, NJ dendrogram and PCOA, PCA, PCO plot produced similar results Therefore, only NJ dendrogram is presented here (Fig. 2). Almost populations of each species were located close to each other. This indicates that the ISSR molecular marker can be used to determine the species delimitation of Tragopogon. ISSR determine delimitation of species and show relationship between species in this genus. ISSR can determine delimitation of species and relationship between species in this genus. Two populations of $T$. gaudanicus with species of T. jesdianus, T. porphyrocephalus, T. bakhtiaricus, T. montanus and T. caricifolius are in one main cluster. Populations of T. vvedenskyi, T. vaginatus, T. graminifolius are located in separated cluster. Species of T.kotschyand T. marginatus are showed close relationship. Populations of T. longirostris are located in one cluster and are closely related to T. gongylorrhizus. A number of T. buphthalmoides populations and T. bornmuelleri populations are show close relationship. All populations of $T$. kemulariae were placed in a cluster. The Evanno test produced delta $\mathrm{k}=7$ as the best number of genetic groups. The STRUCTIRE plot based on k= 7 (Fig. 3) identified seven genetic groups/gene pools. The genetic affinity revealed by STRUCTURE analysis was almost in agreement with the NJ tree result. 


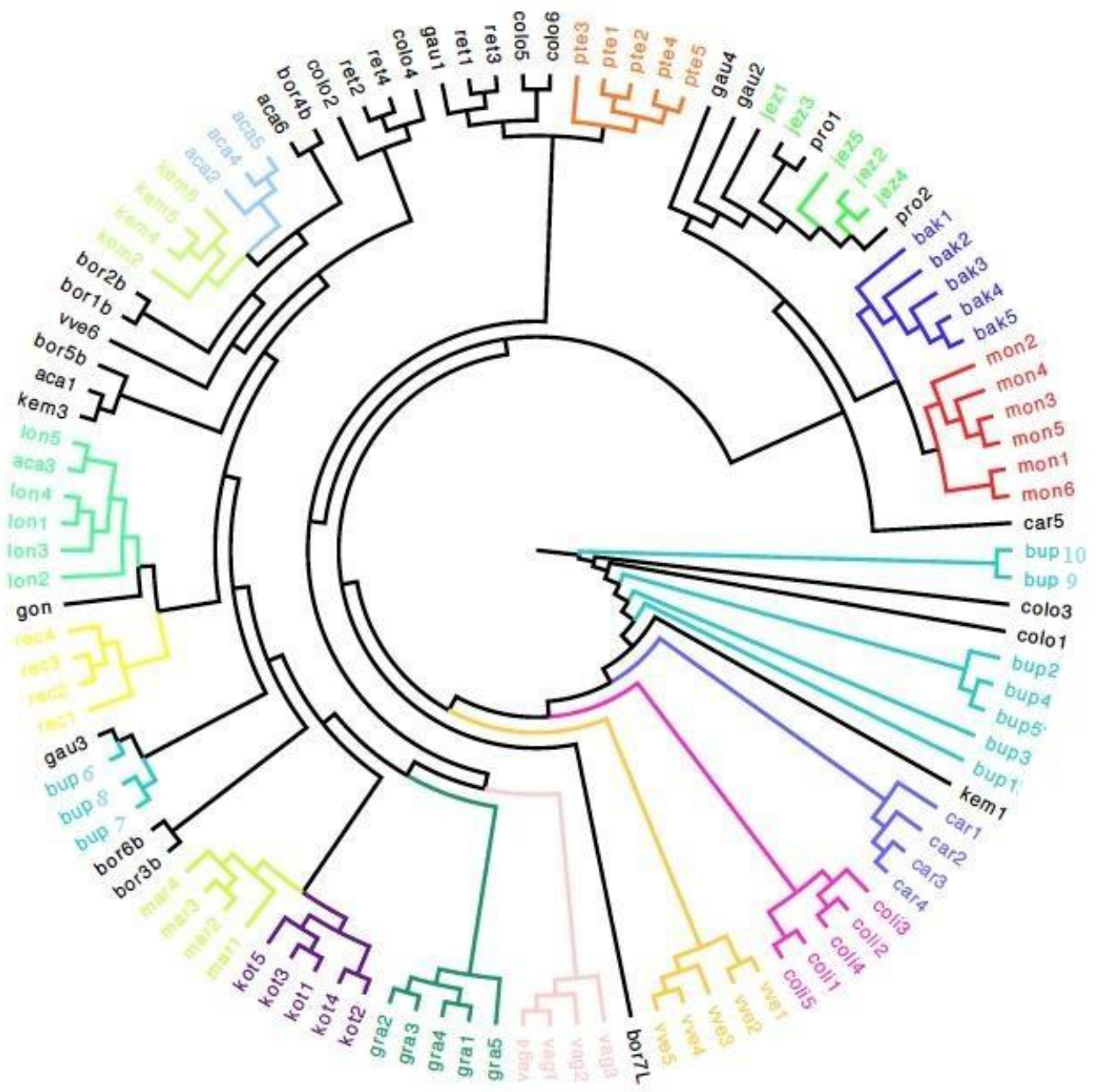

226

227

228 


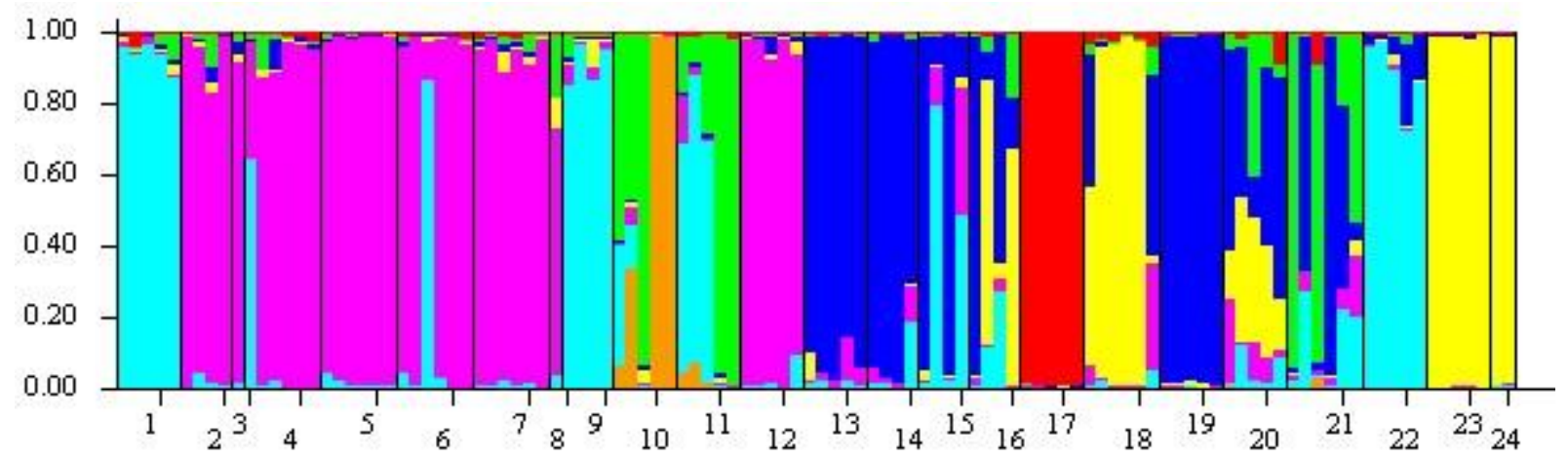

Fig 3. STRUCTURE plot of Tragopogon species based on ISSR

1:T. graminifolius, 2: vaginatus, 3: T. gongylorrhizus, 4: T. vvedenskyi, 5: T. kemulariae, 6: T. acanthocarpus, 7: T. bornmuelleri var. bornmuelleri, 8: T. bornmuelleri var. latifolius, 9: T. rechingeri, 10: T. buphthalmoides var. buphthalmoides, 11: T. buphthalmoides var. latifolius, 12: T. longirostris, 13: T. kotshy, 14: T. marginatus, 15: T. reticulatus, 16: gaudanicus, 17: T. bakhtiariccus, 18: T. montanus, 19: T. collinus, 20: T. caricifolius, 21: T. coloratus, 22: T. pterocarpus, 23: T. jesdianus, 24: T. porphyrocephalus.

ITS and Cp-DNA sequences

NJ, Maximum parsimony and maximum likelihood trees produced similar results and therefore ML tree is presented and discussed (Fig 4). The ML tree based on ITS has two main clusters, T. bornmuelleri var. latifolius is separated from the rest of the species and second cluster is divided into two sub-clusters, One of the sub-clusters includes $T$. buphthalmoides and T. acanthocarpus. The rest of the species are arranged in sub clusters. T.gaudanicus,T.montanus, T. collinus, T. caricifolius, T. jesdianus, T. stroterocarpus, T. porphyrocephalus, T. bakhtiaricus, T. graminifolius, $T$. rechingeri, T. bornmuelleri, T. kotschy and T. marginatus are in one sub-cluster and T. rezaiyensis, T. longirostris, T. pterocarpus, T. coloratus, T. vvedenskyi, T. kemulariae are in one sub-cluster. The tree based on cp DNA datashowed poor clustering (Fig 5). T. marginatus, T. colinus and T. rechingeri are arranged in one cluster. Cp DNA data are not suitable for classifying and examining relationship between species in this genus, The results based on ITS sequences are more efficient than the results based on cp DNA for species delimitation and relationship between species. 


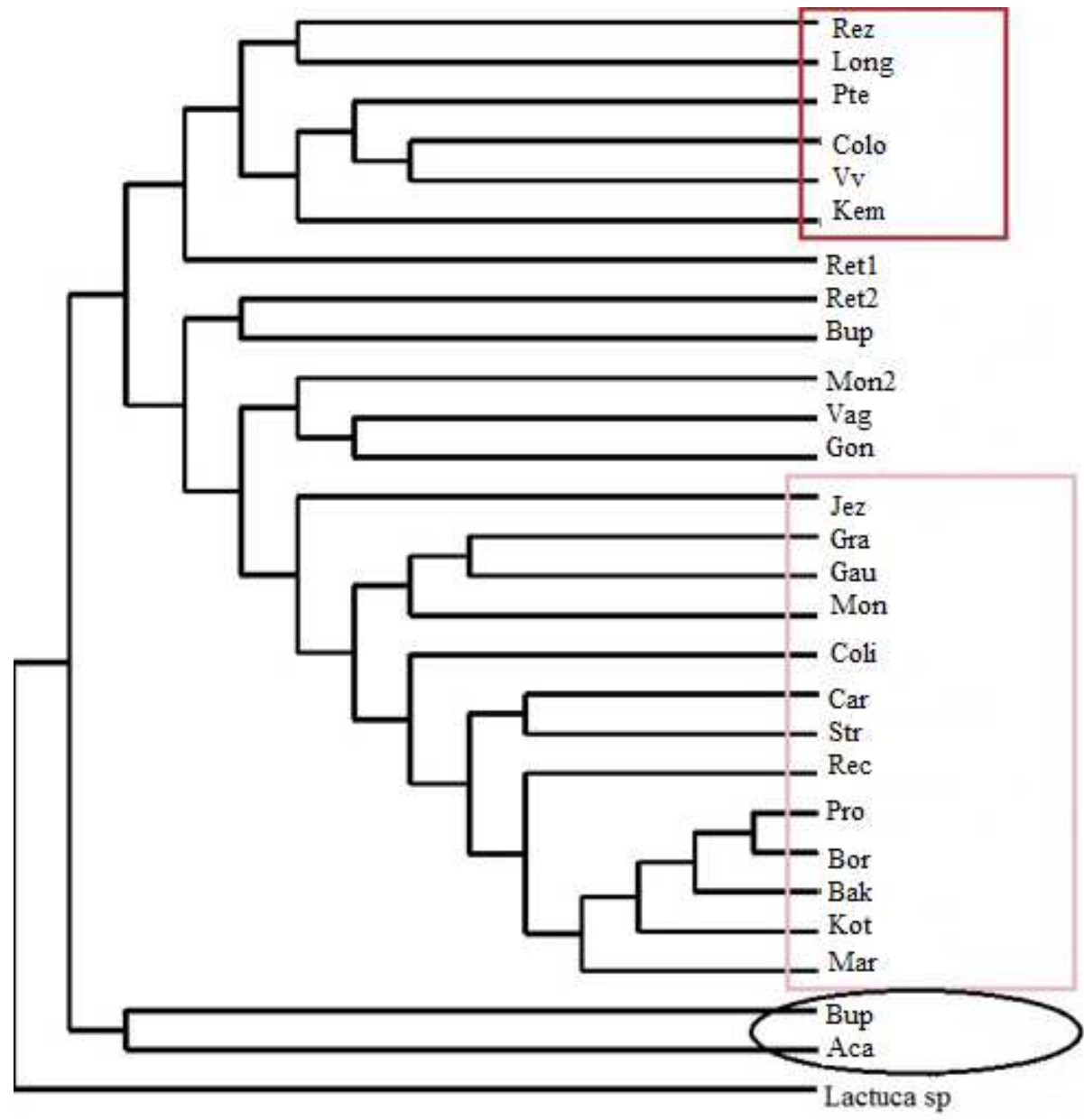

Fig 4. ML tree based on ITS sequence data in Tragopogon genus (Lactuca sp: out group, Species code are according 265 to Table 1). 
Fig 5. ML tree based on cp DNA data in Tragopogon genus (Lactuca sp: out group, Species code are according to Table 1).

\section{DISCUSSION}

293 Based on the morphological studies, the species related to each section were placed close to each other and the border 294 between the sections is almost clear. Species of section Majores (T. capitatus, T. vaginatus, T. afghanicus, T. 295 paradoxus) section of Kemulariae (T. kemulariae) section of Krascheninnikovia (T. longirostris) section of 296 Tuberosi(T. gongylorrhizus )section of Tragopogon( T. gracilis, T. badachschanicus ) section of Angustissimi( (T. 297 sosnovsky, T. vvedenskyi) are clustered based on morphometric data and correspond to the sections of Iranica flora . 298 Marodive et al. 2005 have proven that Tragopogon and Majores sections are monophilic based on ITS-ETS data. 299 Sections include Kemulariae (T. kemulariae), Krascheninnikovia (T. longirostris) Tuberosi (T. gongylorrhizus) 
confirmed based on ISSR data. Majores, Angustissimi, Krascheninnikovia and Tuberosi sections have been confirmed based on morphometric and ISSR studies, which are consistent with studies by Azizi et al. 2021 and Iranica flora.

Species of T. kotschy, T. marginatus, T. reticulatus and T. maturatus are introduced according to the flora of Iranica in Sosnowskya section, which in the present morphometric studies, except for T. maturatus, are arranged in a cluster. Based on morphometric data of T. collinus, T. caricifolius, T. bakhtiaricus, T. gaudanicus and T. montanus are in the same cluster and correspond to section Rubriflori in the flora of Iranica.

T. buphthalmoides, T. bornmuelleri, T. rechingeri, T. acanthocarpus, T. graminifolius, T. reticulatus are based on morphometric data in a cluster, Species of T. buphthalmoides, T. bornmuelleri, T. rechingeri, T. acanthocarpus, are classified in the profundisulcate section based on flora of Iranica. T. graminifolius is classified in the Brevirostres section and T.reticulatus is classified in the Sosnowskya section in the flora of Iranica. Based on morphometric data, two species T. graminifolius and T. reticulatus are in section profundisulcati. Subsequently, based on studies of seed morphology and morphology by Sukhorukov and Nilova (2015) and molecular studies of ITS, ETS by Mavrodiev et al. (2005) has also been confirmed. Two species of T. pterocarpus and T. coloratus are located in two separate clusters close to each other based on morphometric data and ISSR data. According to the flora of Iranica, species of $T$. coloratus and T. petrocarpus have many similarities to each other and are located in the section of Chromopapus Pollen studies performed by Azizi et al. (2021) also confirm this section, therefore We confirm the chromopapus section.

Rubriflori sections based on morphological, ISSR, ITS data confirms the flora of Iranica and pollen studies by Azizi et al. (2021).Classification of Iranian endemic species such as T. jesdianus, T. erostris, T. porphyrocephalus, $T$. rezaiyensis, T. stroterocarpus in the flora of Iranica is unknown, Marodive 2012 Based on 7 nuclear loci (Adh, GapC, LFY, AP3, PI, ITS, ETS) studies species of T. rezaiyensis in B clade, species of T.jesdianus in C clade, species of $T$. stroterocarpus in D clade, species of T. porphyrocephalus in F clade classified. Based on our morphometric studies, these species belong to the Rubriflori section, ISSR data also proves that two species of T. prophyrocephalus and T. jesdianus belong to the Rubriflori section. Rubriflori section introduced in Iranica flora has been proved by morphometric and ISSR data. The species T.porphyrocephalus, T. stroterocarpus and T. jesdianus belong to Rubriflori section based on ITS data results which is consistent with the results of pollen data by Azizi et al. (2021). 
According to Marodive et al. (2005) studies, T. jesdianus species were classified in Collini (Rubriflori) section, which this section has T. montanus, T. bornmuelleri, T. collinus, T. jesdianus, T. marginatus.

Populations of T. vaginatus species in one cluster as well as populations of $T$. vvedenskyi species in one cluster and populations of T. graminifolius species in one cluster are arranged, Each of these species, according to the flora of Iranica, are placed in separate sections under the names of Majores, Angustissimi, Brevirostris, respectively The ISSR data results confirm these three sections.

According to the ISSR data, populations of T. longirostris are located in a cluster and confirm the Krascheninnikovi section of the flora of Iranica.

According to ITS data, T. rezaiyensis, $T$. longirostris, T. ptrocarpus, T. coloratus, T. vvedenskyi, T. kemulariae are related to each other Which is almost consistent with clade B by Marodive et al. 2012 studies based on 7 nucleargenes (Adh, GapC, LFY, AP3, PI, ITS, ETS).

According to ITS data, T. buphthalmoides and T. acanthocarpus species are located in a cluster that confirms the Profundisulcati section of Iranica flora.

Cp DNA dendrogram are not useful for classification in this genus and Chloroplast sequences are very similar among Tragopogon species, Therefore, the use of cp DNA markers in the classification of this genus is not recommended.

\section{Compliance with ethical standards}

Confl ict of interest The authors declare that they have no conflict of interest.

\section{References}

Azizi H, Sheidai M, Mozaffarian V, Noormohammadi Z (2021), pollen morphology of the genus Tragopogon (Asteraceae). Acta Bot. Hung. 63(1-2):31-43.

https://doi.org/ 10.1556/034.63.2021.1-2.2.

Bell CD, Mavrodiev EV, Soltis PS, Calaminus AK, Albach DC, Cellinese N, Garcia-Jacas N, Soltis DE (2012) Rapid diversification of Tragopogon and ecological associates in Eurasia. J. Evol. Biol. 25: 2470-2480. //doi.org/10. 10.1111/j.1420-9101.2012.02616.x 
Blanca G, Diazdela Guardia C. (1997). Fruit morphology in Tragopogon L. (Compositae: Lactuceae) from the Iberian Peninsula. Bot. J. Linn. 125:319-329.

357

358

359

360

361

362

363

364

365

366

367

368

369

370

371

372

373

374

375

376

377

378

379

380

381

Buggs RJA, Soltis PS, Mavrodiev EV, Symonds VV, Soltis DE (2008). Does phylogenetic distance between parental genomes govern the success of polyploids Castanea 73(2): 74-93.http://dx.doi.org/10.2179/0008-7475 (2008)73[74: DPDBPG] 2.0.CO;2.

Falush D, Stephens M, Pritchard JK (2007). Inference of population structure using multilocus genotype data: dominant markers and null alleles. Mol. Ecol. Notes 7:574-578.

Hamer Q, Harper DA, Rayan PD (2012).PAST: Paleontological Statistics software package for education and data analysis. Palaeontol. Electron. 4: 9.

Kashin AS, Berezutsky MA, Kochanova IS, Dobrynicheva NV, Polyanskaya MV (2007). Pecularities of seed reproduction in populations of Asteraceae species under impact of anthropogenic factors. Botanicheskiy Zhurnal 92(9): 1408-1427. [In Russian]

Mavrodiev EV, Edwards CE, Albach DC, Gitzendanner A, Soltis PS, Soltis DE (2004)"Phylogenetic relationships in subtribe Scorzonerinae (Asteraceae: Cichorioideae: Cichorieae) based on ITS sequence data" Taxon 3: 699-712.

Mavrodiev EV, Tancig M, Sherwood AM, Gitzendanner MA, Rocca J, Soltis PS, Soltis DE (2005) Phylogeny of Tragopogon L. (Asteraceae) based on internal and external transcribed spacer sequencedata. International Journal of Plant Sciences 166: 117-133.

Mavrodiev EV, Soltis PS., Soltis DE (2008a), Putative parentage of six Old World polyploids in Tragopogon L. (Asteraceae: Scorzonerinae) based on ITS, ETS and plastid sequence data. Taxon 57: 1215-1232.

Mavrodiev EV, Nawchoo I, Soltis PS, Soltis DE (2008b), Molecular data reveal that the tetraploid

Tragopogon kashmirianus (Asteraceae: Lactuceae) is distinct from the North American T. mirus. Bot. J. Linn. 158: 391-398.

Mavrodiev EV, Albach DC, Speranza P (2008c) Anew polyploid species of the genus Tragopogon (Asteraceae, Cichorieae) from Russia. Novon 18: 229-232.

Ownbey M (1950) Natural hybridization and amphiploidy in the genus Tragopogon. Am. J. Bot. 37(7): 487-499. //doi.org/10.2307/2438023. 
Ownbey M, McCollum GD (1953) Cytoplasmic inheritance and reciprocal amphiploidy in Tragopogon. Am. J. Bot.

Peakall R, Smouse PE (2006). GENALEX 6: genetic analysis in Excel. Population genetic software for teaching and research. Mol. Ecol. Notes. 6: 288-295.

Pires JC, Lim KY, Kovarik A, Matyasek R, Boyd A, Leitch AR, Leitch IJ, Bennett MD, Soltis PS, Soltis DE. (2004) Molecular cytogenetic analysis recently evolved Tragopogon (Asteraceae) allopolyploids reveal a karyotype that is additive of the diploid progenitors. Am. J. Bot. 91: 1022-1035.//doi.org/10.3732/ajb.91.7.1022.

401

Podani J (2000) Introduction to the Exploration of Multivariate Data. Backhuys Publ, Leiden.

Pritchard JK, Stephens M, Donnelly (2000) Inference of population structure using multilocus genotype Data.

Rechinger KH (1977) “Tragopogon” In: Rechinger KH (ed) Flora Iranica, Compositae II, Lactuceae. Osterreich:

Richardson I B K (1976) "Tragopogon" In: Tutin TG, Heywood V H, Burges NA, Moore DM, Valentine DH,

Walters S A, Webb D A (eds) Flora Europaea, vol. 4. Cambridge Univ. Press, Cambridge.

Safavi S R, Naseh Y, Jafari E, Tavakoli Z, Heidar N (1992) Flora Iran, (Asteraceae Tribe cichorieae), Research

Schishkin BK, Bobrov EG (1961) Compositae Tragopogon in KomarovFlora URSS. Doon Scientific Translation co. 318, Chukhuwala, Dehra Dun, India, 29: 131-196.

Shaw J, Lickey EB , Beck JB, Farmer SB, Lium W, Miller J, Siripun KC (2005) The tortoise and the hare II:

Sheidai M, Zanganeh S, Haji- Ramezani R, Nouroozi M, Noormohammadi Z, Ghasemzadeh- Baraki S (2013) 
428 Tamura K, Peterson D, Peterson N, Stecher G, Nei M, Kumar S (2011). MEGA5: Molecular Evolutionary Genetics 429 Analysis using Maximum Likelihood, Evolutionary Distance, and Maximum Parsimony Methods. Mol. Biol. Evol. $430 \quad 28: 2731-2739$.

431 Timme R, Kuehl EJ, Boore JL, Jansen RK (2007) Acomparative analysis of the Lactuca and Helianthus

432 (Asteraceae) plastid genomes: identification of divergent regions and categorization of shared repeats. Am. J. Bot.

433 94: 302-313.

434

435 White TJ, Bruns S, Lee S, Taylor J (1990) Amplification and direct sequencing of fungal ribosomal RNA genes for 436 phylogenetics. In: Innis MA, Gelfand DH, Sninsky JJ, White TJ (eds) PCR Protocols: 315-322 Academic Press, 437 London. 